\title{
EDITORES CHEFE
}

Dra. Ana Paula Perfetto Demarchi

Dr. Rogério Zanetti Gomes

\section{CONSELHO EDITORIAL}

Dra. Ana Luisa Boavista Lustosa Cavalcante, Universidade Estadual de Londrina - UEL Dra. Lucimar de Fátima Bilmaia Emidio, Universidade Estadual de Londrina - UEL Dr. Marcelo Castro Andreo, Universidade Estadual de Londrina - UEL Me. Paula Hatadani, Universidade Estadual de Londrina - UEL Dr. Rogério Zanetti Gomes, Universidade Estadual de Londrina - UEL Dra. Rosane Fonseca de Freitas Martins, Universidade Estadual de Londrina - UEL Dra. Vanessa Tavares de Oliveira Barros, Universidade Estadual de Londrina - UEL

CONSELHO CONSULTIVO AD HOC

Dra. Ana Mery Sehbe De Carli - UFSC - Brasil PhD Bernabé Hernandis Ortuño - Universidad Politecnica de Valencia - Espanha PhD. Brigitte Borja de Mozota - Parsons Paris e Universit Paris Quest - França

Dr. Cláudio Freitas de Magalhães - PUC - Rio - Brasil

PhD. Dijon Moraes Júnior - UFMG - Brasil

Dr. Eugenio Andrés Díaz Merino - UFSC - Brasil

Dra. Evelise Anicet Rüthschilling - UFRGS - Brasil

Dr. Francisco Antonio Pereira Fialho - UFSC - Brasil

Dra. Giselle Beiguelman - FAU USP - Brasil

Dra. Kathia Castilho - Anhembi Morumbi - Brasil

Dra. Laura Bezerra Martins - UFPE - Brasil

Dra. Lia Buarque de Macedo Guimarães - UFRS - Brasil

Dra. Lucy Niemeyer - ESDI - Brasil e IADE/ Universidade Europeia, Portugal

Dra. Marilda Lopes Pinheiro Queluz - UTFPR - Brasil PhD Mário Duarte de Araújo - Universidade do Minho - Portugal

Dra. Maristela Mitsuko Ono - UFPR - Brasil Dra. Virginia Kistmann - UFPR- Brasil 


\section{EDITORA TÉCNICA}

Laudicena Ribeiro

NORMALIZAC̣̃̃O

Natali Silvana Zwaretch

REVISOR DE LINGUA INGLESA

Murilo Crivellari Camargo

\section{PROJETO GRÁFICO}

Gabriel Darcin Alsouza

EDITORAC̣ÃO

Murilo Crivellari Camargo

\section{AVALIADORES NESTE NÚMERO}

Ana Mery De Carli

Cleuza Bittencourt Ribas Fornasier

Cristiane Affonso de Almeida Zerbetto

Juliana Barbosa

Livia Pereira

Lucimar Bilmaia Emidio

Marcelo Castro Andreo

Maria Celeste Sanches

Patrícia de Mello Souza

Renato Domingues Záccaro Macri

Rosane Fonseca de Freitas Martins

Suraia Farah

Thassiana de Almeida Miotto Barbosa

Viviane Aiex

Catalogação elaborada pela Divisão de Processos Técnicos da Biblioteca Central da Universidade Estadual de Londrina

Dados Internacionais de Catalogação-na-Publicação (CIP)

Projética [recurso eletrônico] / Universidade Estadual de Londrina, Centro de Educação, Comunicação e Artes, Departamento de Design. - V.1, n.1 (dez. 2010)- . Londrina: UEL, 2010-

Disponível em: http://www.uel.br/revistas/uel/index.php/projetica Semestral.

ISSN 2236-2207

1. Design. 2. Gestão de produtos e tecnologia. 3. Ergonomia e usabilidade. 4. Design de moda. 5. Design Digital. 6. Design Gráfico. I. Universidade Estadual de Londrina. Centro de Educação Comunicação e Artes. Departamento de Design. 